\title{
Mastectomía endoscópica por puerto único: técnica quirúrgica y primer caso en México
}

\author{
Single port endoscopic mastectomy: surgical technique and first case in Mexico
}

Guillermo G. Peralta-Castillo ${ }^{1,2 *}$ Ricardo Cavazos-García ${ }^{3,4}$, Eva Eulalia-Hernández ${ }^{1,2}$, Luis M. Cornejo-Mota ${ }^{5}$ y Ana C. Santiago-Prieto ${ }^{6}$

${ }^{1}$ Clínica de Mama, Cancer Center Tec 100, Querétaro, Querétaro; 2Departamento de Cirugía General, Hospital H+, Querétaro, Querétaro; ${ }^{3}$ Departamento de Cirugía, Hospital Ángeles del Carmen, Guadalajara, Jalisco; ${ }^{4}$ Escuela de Ciencias de la Salud y Medicina, Universidad del Valle de México, Campus Zapopan, Jalisco; ${ }^{5}$ Departamento de Cirugía Plástica, Hospital H+ Querétaro, Querétaro; ${ }^{6}$ Departamento de Patología Quirúrgica, Hospital H+, Querétaro, Querétaro. México

\section{Resumen}

Las innovaciones técnicas han permitido realizar un sinnúmero de cirugías endoscópicas y laparoscópicas, entre ellas la mastectomía preservadora de piel y pezón por puerto único, que ha sido reportada como bien tolerada, con un excelente resultado cosmético y con el mismo pronóstico oncológico que la cirugía tradicional. Esta técnica, común en Asia, presenta diversos retos técnicos y económicos. Presentamos la primera aproximación en nuestro país sobre este abordaje en una paciente con cáncer de mama. Para realizar esta cirugía es preciso el entrenamiento en mínima invasión y en cirugía mamaria, así como una adecuada selección de las pacientes.

Palabras clave: Cáncer de mama. Endoscopia. Mastectomía. Puerto único.

\begin{abstract}
Technical innovations and scientific advances have allowed to perform countless endoscopic and laparoscopic surgical procedures. Among these, Single port endoscopic Mastectomy has been reported as well tolerated, with great aesthetic results and the same oncologic prognosis as traditional surgery. This popular approach in Asian countries presents many technical and economic challenges. We present the first endoscopic approach in our country: a female patient with infiltrating ductal carcinoma in the left breast. We understand that to carry out this procedure, adequate patient selection, great minimal invasive and breast surgery skills are necessary.
\end{abstract}

Key words: Breast cancer. Endoscopy. Mastectomy. Single port.

\section{Introducción}

Desde finales del siglo XIX ha habido avances muy significativos en el tratamiento quirúrgico del cáncer de mama, desde la cirugía radical de William Hasted y sus modificaciones por Patey y Dyson hasta el abordaje conservador propuesto por Veronessi ${ }^{1-3}$. Así, en la búsqueda de realizar cirugías menos invasivas, y

Correspondencia:

*Guillermo G. Peralta-Castillo

Prol. Priv. Ignacio Zaragoza, 263 Centro

Fecha de recepción: 04-10-2019

Fecha de aceptación: 31-07-2020

DOI: $10.24875 / C I R U .19001631$
Cir Cir. 2020;88(S2):108-112

Contents available at PubMed www.cirugiaycirujanos.com

E-mail: drperalta-g@outlook.com 0009-7411/@ 2020 Academia Mexicana de Cirugía. Publicado por Permanyer. Este es un artículo open access bajo la licencia CC BY-NC-ND (http://creativecommons.org/licenses/by-nc-nd/4.0/). 
junto con los avances en cirugía plástica, fue introducida la mastectomía preservadora de piel y pezón como un abordaje oncológicamente seguro, bien tolerado y con excelentes resultados estéticos ${ }^{4}$. En la última década, este abordaje se ha vuelto muy común en todo el mundo, pues se puede realizar en cirugías profilácticas o terapéuticas, con un adecuado resultado cosmético ya que preserva la totalidad de la «envoltura de la mama».

Dándole continuidad a esta idea, y con el objetivo de la completa erradicación del cáncer y el intento de preservar la anatomía de la paciente, en 2002 surge la cirugía endoscópica de mama, aunque anteriormente había sido aplicada en la disección axilar ${ }^{5,6}$. La cirugía endoscópica y laparoscópica ha ganado numerosos adeptos y hoy en día es considerada el estándar de tratamiento en muchas patologías. En cuanto a la cirugía mamaria, ha ganado popularidad, en especial en países asiáticos en las últimas dos décadas, principalmente porque el tamaño de la mama en los países orientales es menor y realizar una cirugía conservadora con buenos resultados estéticos no siempre es posible ${ }^{7-9}$. Además, en los últimos años, la cirugía endoscópica de mama ha ganado un nuevo impulso en Occidente con el abordaje robótico de Toesca en el Instituto Europeo de Oncología ${ }^{10}$. Sin embargo, algunas de estas técnicas han sido abandonadas debido a su complejidad, por lo que se considera fundamental tener un adecuado entrenamiento laparoscópico y mamario para realizar este tipo de abordaje $e^{11,12}$.

\section{Caso clínico}

Mujer de 63 años, con una tumoración de $2.9 \mathrm{~cm}$ en la mama izquierda de 3 meses de evolución, con diagnóstico mediante biopsia por aguja de corte de adenocarcinoma ductal infiltrante, clínicamente sin actividad ganglionar, en etapa clínica IIA. Presenta una mama pequeña, con una mala relación mama-tumor para considerarla para cirugía conservadora. La inmunohistoquímica corresponde a un tumor luminal A, por lo que, en sesión multidisciplinaria, se decide candidata a mastectomía total preservadora de piel y pezón más biopsia de ganglio centinela y primer tiempo de reconstrucción, cirugía que se lleva a cabo previo consentimiento informado.

Durante el procedimiento se realiza una incisión de $3 \mathrm{~cm}$ en la línea axilar anterior, asegurándonos de que pueda quedar oculta por el brazo. Se realiza una disección subcutánea, creando un colgajo de $4 \mathrm{~cm}$ bajo

\section{A}
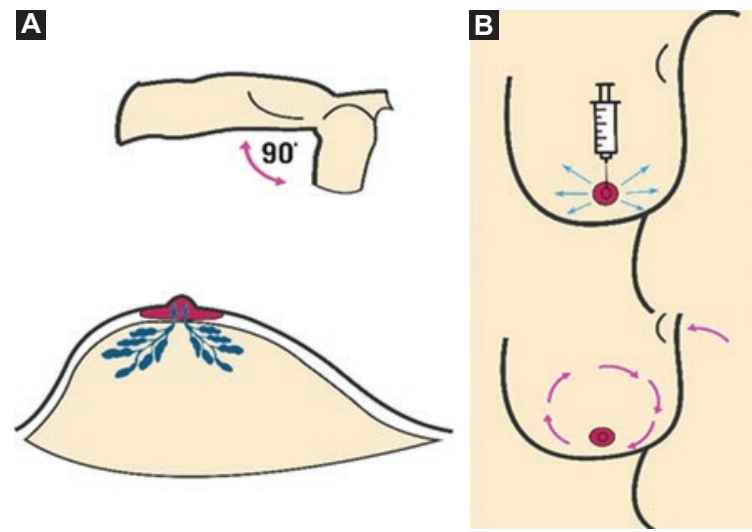

Figura 1. A: con la paciente en decúbito dorsal y el brazo extendido a $90^{\circ}$ se planean la incisión y la colocación del puerto único en una incisión axilar para asegurar que se oculta por el brazo de la paciente. B: la disección comienza con la incisión axilar y la posterior hidrodisección e inyección subcutánea de solución salina (que contiene lidocaína y epinefrina) ocupando la totalidad de la mama para reducir el sangrado. C: inicialmente se inserta el puerto único y se comienza con la disección endoscópica del colgajo superior. Una vez que se alcanza la porción posterior del pezón, se identifica con azul patente (por la inyección del ganglio centinela). D: se continúa con la disección periférica hasta crear el espacio suficiente para disecar la glándula del pectoral mayor.

visión directa, y se procede a realizar ganglio centinela con doble técnica (azul patente y radio nucleótido). Posteriormente se instila solución salina con lidocaína al $0.05 \%$ y epinefrina $(1: 1,000,000)$ de manera subcutánea y radial al pezón para disminuir el riesgo de sangrado. Una vez que se obtiene un espacio adecuado para trabajar, se inserta el puerto único, conectado a un insuflador y manteniendo la presión a $7 \mathrm{mmHg}$. La disección se realiza con cauterio monopolar y tijera de $5 \mathrm{~mm}$. Se usan tracción y contratracción para mantener una adecuada exposición con pinza de Maryland de $5 \mathrm{~mm}$ y tracción digital del pezón por el ayudante. La disección comienza en el colgajo superficial, por los cuadrantes externos, hasta llegar al pezón. Se toman dos muestras de la porción posterior del pezón y se mandan a análisis citopatológico transoperatorio. Se continúa con la disección anterior por completo hasta crear suficiente espacio para seguir con la disección posterior sobre el pectoral mayor. La glándula fue extirpada en bloque por la incisión axilar de $3 \mathrm{~cm}$. Los bordes del tumor se valoraron en el transoperatorio por el departamento de patología (Figs. 1 y 2).

En la etapa reconstructiva, la presión de gas no fue suficiente para ayudar a elevar el pectoral mayor, por lo que se retiró el puerto y se usó un retractor estándar para la disección y la colocación del expansor. 

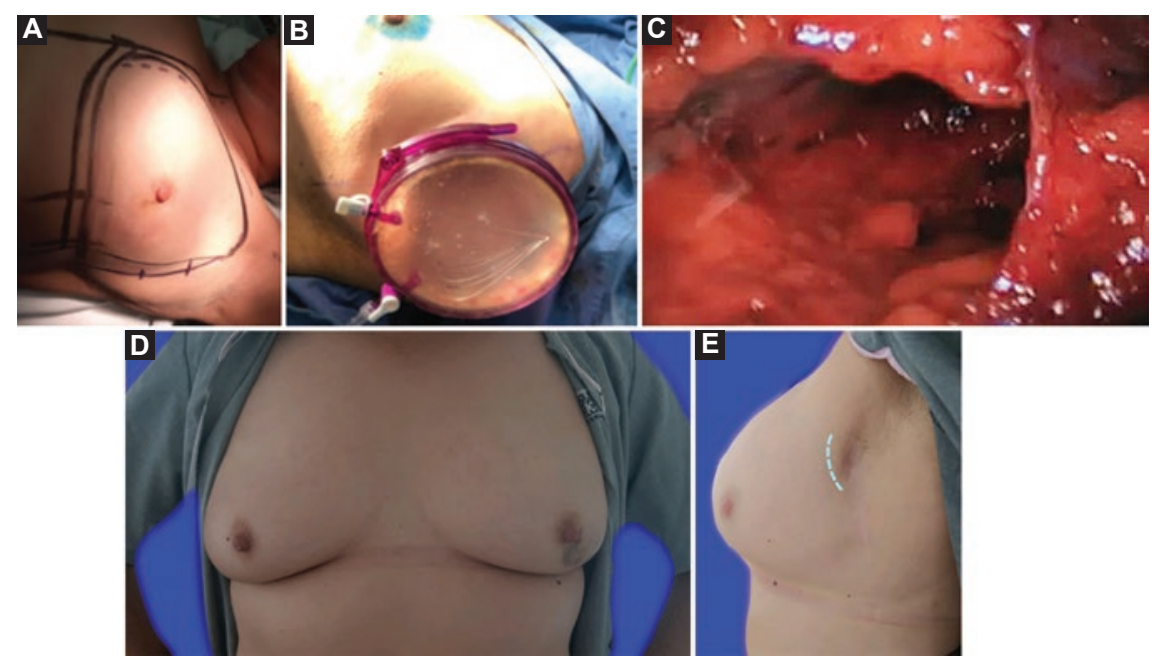

Figura 2. Técnica quirúrgica. A: planeación de la cirugía. B: incisión axilar de $3 \mathrm{~cm}$ y colocación del puerto único. C: disección anterior hasta llegar al complejo areola-pezón. D: imagen prequirúrgica. E: imagen en la semana 6 posoperatoria con $390 \mathrm{~cm}^{3}$ en el expansor.

El tiempo quirúrgico total de la disección oncológica fue de 140 minutos (incluidos los estudios transoperatorios del ganglio centinela y la zona retroareolar) y el sangrado transoperatorio fue de $80 \mathrm{~cm}^{3}$. La paciente fue egresada al segundo día posquirúrgico sin complicaciones. Actualmente se encuentra con un periodo libre de enfermedad de 12 meses, es decir, no presenta datos clínicos ni radiológicos de recaída local o a distancia. Además, cuenta con el mejor resultado $(16 / 16$, altamente satisfecha con el procedimiento quirúrgico) en la subescala de satisfacción del test BRECON-31 13 .

\section{Discusión}

Mantener un aspecto natural de la mama con una adecuada preservación del complejo areola-pezón disminuye el impacto psicológico en una paciente mastectomizada ${ }^{14}$, y realizar una incisión que no afecte la sensibilidad del pezón y promueva un mejor resultado estético siempre será un reto.

Reportamos el primer caso de mastectomía subcutánea preservadora de piel y pezón endoscópica en México. Sabemos que la mastectomía endoscópica es una técnica quirúrgica segura, con una baja morbilidad perioperatoria, una tasa baja de isquemia y necrosis del pezón, y un adecuado resultado estético. En la literatura se han reportado diversos abordajes endoscópicos, a través de incisiones periareolares o axilares, y han demostrado ser una alternativa efectiva, bien tolerada y oncológicamente segura para la resección de tumores malignos ${ }^{15-21}$, con o sin reconstrucción inmediata, siendo reportadas como cirugías con un excelente resultado estético ${ }^{15,22}$.

Debido a la característica propia de la mastectomía, que se define como mutilante, comúnmente se asocia a problemas psicológicos, como depresión, estrés, ansiedad y dificultades para llevar a cabo relaciones interpersonales ${ }^{23-25}$. Estos efectos pueden disminuirse con una adecuada reconstrucción, en especial en pacientes con cirugías preservadoras de piel y pezón $^{14,26,27}$, por lo que esta técnica brinda otra herramienta de tratamiento para las pacientes con cáncer de mama.

Existen diversos reportes de la seguridad oncológica de esta técnica ya desde sus inicios en 2002, con trabajos que demostraron que no hay diferencias significativas en cuanto a pronóstico, sobrevida, recurrencia local y metástasis a distancia ${ }^{11,12,28}$. Hasta el momento, las principales indicaciones de la mastectomía endoscópica incluyen ${ }^{9}$ :

- Carcinoma ductal in situ.

- Tumores pequeños (T1 y T2) en pacientes con mamas pequeñas en las que la cirugía conservadora dejará obvias deformidades.

- Pacientes candidatas a cirugía conservadora que no desean (o no puedan recibir) radioterapia y optan por la mastectomía.

- Pacientes que desean cirugía reductora de riesgo. Los principales retos que afronta esta técnica son la adecuada selección de pacientes, el tiempo quirúrgico y los costos mayores en comparación con las técnicas tradicionales, motivos por lo que pensamos 
que ha carecido de popularidad. Sin embargo, en nuestra experiencia, el tiempo quirúrgico oncológico fue de 140 minutos, ligeramente menor que los reportados por Ho, et al. ${ }^{5}$, de 195 minutos, y Hung, et al. ${ }^{29}$, de 228 minutos. Comparado con otras técnicas subcutáneas, no creemos que el tiempo sea un factor tan importante. Además, sabemos que el tiempo quirúrgico disminuye a medida que aumenta el número de $\operatorname{casos}^{29}$.

\section{Conclusiones}

Con los avances actuales en la cirugía endoscópica, las pacientes que son candidatas a mastectomía preservadora de piel y pezón pueden ahora beneficiarse de la cirugía endoscópica, disminuyendo el tamaño de la cicatriz quirúrgica, mejorando la apariencia estética y, tal vez, reduciendo los efectos psicosociales que todo el proceso conlleva.

En cuanto a la técnica, creemos que el uso de dióxido de carbono pareciera habernos permitido una menor cantidad de sangrado, ayudándonos además a respetar y preservar las perforantes de las arterias intercostales, lo que contribuye a una mejor circulación en el pezón. Además, en nuestra opinión, la incisión en la axila puede ser fácilmente ocultada por el brazo, contribuyendo también a la vitalidad del complejo areola-pezón y mejorando la estética de la cirugía.

Sin embargo, reconocemos algunas limitaciones importantes que pudieran influir en la falta de popularidad de esta técnica: los instrumentos utilizados fueron diseñados para otros propósitos y esto dificulta su adecuado funcionamiento; el uso de esta tecnología incrementa los costos y el tiempo de la cirugía, mismos que deberán ser valorados con la paciente previamente a la realización de este abordaje. Además, la selección de las pacientes es importante, ya que los tamaño del tumor y de la mama pueden ser un impedimento para la técnica, y por desgracia en nuestro medio los casos avanzados representan un gran porcentaje de las pacientes afectadas.

Entendemos que es preciso un adecuado entrenamiento laparoscópico o endoscópico y en cirugía mamaria para llevar a cabo este procedimiento. Estamos seguros de que con el incremento en el uso de terapias neoadyuvantes y una mayor difusión de la técnica, esta será considerada con mayor frecuencia en la elección de tratamientos quirúrgicos oncológicos en la mama.

\section{Agradecimientos}

Los autores agradecen a la Dra. Denisse Sepúlveda por la revisión del artículo, a la licenciada Lourdes Rangel por su ayuda durante la cirugía y al Hospital San José de Querétaro por las facilidades otorgadas en sus instalaciones.

\section{Conflicto de intereses}

Ninguno de los autores presenta conflicto de intereses.

\section{Responsabilidades éticas}

Protección de personas y animales. Los autores declaran que para esta investigación no se han realizado experimentos en seres humanos ni en animales.

Confidencialidad de los datos. Los autores declaran que han seguido los protocolos de su centro de trabajo sobre la publicación de datos de pacientes.

Derecho a la privacidad y consentimiento informado. Los autores han obtenido el consentimiento informado de los pacientes y/o sujetos referidos en el artículo. Este documento obra en poder del autor de correspondencia.

\section{Bibliografía}

1. William $\mathrm{H}$. The results of operations for the cure of cancer of breast performed at the Johns Hopkins Hospital from June 1889 to January 1894. Arch. Surg. 1894;20:497-555.

2. Patey DH, Dyson WH. Prognosis of carcinoma of the breast in relation to the type of operation performed. Br J Cancer. 1948;2:7-13.

3. Veronesi U, Saccozzi R, Greco M, Luini A, Sultan L. Conservative treatment of breast cancer. A trial in progress at the Cancer Institute of Milan. Bull Cancer. 1977;64:619-22.

4. Sakamoto N, Fukuma E, Higa, K, Ozaki S, Sakamoto M, Abe S, et al. Early results of an endoscopic nipple sparing mastectomy. Indian J Surg Oncol. 2010;1:232-9.

5. Ho W, Ying S, Chan A. Endoscopic-assisted subcutaneous mastectomy and axillary dissection with immediate mammary prosthesis reconstruction for early breast cancer surgical endoscopy. 2002;16:302-6. doi: https://doi.org/10.1007/s004640000203

6. Salvat J, Knopf JF, Ayoubi JM, Slamani L, Vincen-Genod A, Guilbert M, et al. Endoscopic exploration and lymph node sampling of the axilla. Preliminary findings of a randomized pilot study comparing clinical and anatomo-pathologic results of endoscopic axillary lymph node sampling with traditional surgical treatment. Eur J Obstet Gynecol Reprod Biol. 1996;70:165-73.

7. Yau TK, Soong IS, Sze H, Choi CW, Yeung MW, Ng WT, et al. Trends and patterns of breast conservation treatment in Hong Kong: 1994-2007. Int J Radiat Oncol Biol Phys. 2009;74:98-103.

8. Jeffrey G, Groot G, Boden C, Busch A, Holtslander L, Lim H. Review of factors influencing women's choice of mastectomy versus breast conserving therapy in early stage breast cancer: a systematic review. Clin Breast Cancer. 2018;18:e539-54.

9. Mohammed-Keshtgar RS, Fukuma E. Endoscopic mastectomy: what does the future hold? women's health. 2009;5(2):107-9.

10. Toesca A, Peradze N, Galimberti V, Manconi A, Gentilini O, Sances D, et al. Robotic nipple sparing mastectomy and immediate breast reconstruction with implant: first report of surgical technique. Ann Surg. 2017;266:e28-e30 
11. Leff DR, Vashisht R, Yongue G, Keshtgar M, Yang GZ, Darzi A. Endoscopic breast surgery: where are we now and what might the future hold for the video assisted surgery? Breast Cancer Res Treat. 2011 125:607-25.

12. Tetsuhiro O, Kijima $Y$, Yoshinaka H, Hirata M, Okumura $H$, Ishigami S, et al. Present status of endoscopic mastectomy for breast cancer. World J Clin Oncol. 2015;6:25-9.

13. Temple CF, Cook EF, Bettge-Hahn M, Mychailyshyn N, Naeem H, Madermid J. Development of a breast reconstruction satisfaction questionnaire (BRECON-31): principal components analysis and clinimetric properties. J Surg Oncol. 2012;106:799-806.

14. Sherman KA, Woon S, French J, Elder E. Body image and psychological distress in nipple-sparing mastectomy: the roles of self-compassion and appearance investment. Psychooncology. 2017;26:337-45.

15. Lai HW, Lin SL, Chen ST, Kuok KM, Chen SL, Lin YL, et al. Single axillary incision endoscopic assisted hybrid technique for nipple-sparing mastectomy: technique, preliminary results and patient reported cosmetic outcome from preliminary 50 procedures. Ann Surg Oncol. 2018; 25:1340-9.

16. Lai HW, Chen ST, Chen DR, Chen SL, Chang TW, Kuo SJ, et al. Current trends in and indications for endoscopic assisted breast surgery for breast cancer: results from a six year study conducted by the Taiwan Endoscopic Breast surgery cooperative group. PLoS One. 2016; 11:e0150310

17. Lai HW, Wu HS, Chuang KL, Chen DR, Chang TW, Kuo SJ, et al. Endoscopy-assisted total mastectomy followed by immediate pedicled transverse rectus abdominis musculocutaneous (TRAM) flap reconstruction: preliminary results of 48 patients. Surg Innov. 2015;22:382-9.

18. Lee EK, Kook SH, Park YL, Bae WG. Endoscopy-assisted breast-conserving surgery for early breast cancer. World J Surg. 2006;30:957-64.
19. Sakamoto N, Fukuma E, Higa K, Ozaki S, Sakamoto M, Abe S, et al. Early results of an endoscopic nipple-sparing mastectomy for breast cancer. Indian J Surg Oncol. 2010;1:232-9.

20. Lai HW, Lin HY, Chen SL, Chen ST, Chen DR, Kuo SJ. Endoscopy-assisted surgery for management of benign breast tumors: technique, learning curve, and patient-reported outcome from preliminary 323 procedures. World J Surg Oncol. 2017;15:19.

21. Sakamoto N, Fukuma E, Teraoka K, Hoshi K. Local recurrence following treatment for breast cancer with an endoscopic nipple-sparing mastectomy. Clin Trial. 2016;23:552-60.

22. Wang $Y$, Wu JX, Guan S. A technique of endoscopic nipple-sparing mastectomy for breast cancer. JSLS. 2017;21:e2017.00028.

23. Ominyi JN, Nwodom MU. Psychological impact of mastectomy and breast reconstruction. IJSR. 2014;3:551-6.

24. Alves BT, Melo AMDd, Nascimento SIBd, Sousa Neto JM, Araújo IA, Puentes TMO, et al. The psychological impact in women after mastectomy with and without immediate mammary reconstruction. Am In Mul J. 2018;4:112-6.

25. Ray C. Psychological implications of mastectomy. Br J Soc Clin Psychol. 1977;16:373-7.

26. Dean C, Chetty U, Forrest AP. Effects of immediate breast reconstruction on psychosocial morbidity after mastectomy. Clin Trial. 1983;1:459-62.

27. Rowland JH, Holland JC, Chaglassian T, Kinne D. Psychological response to breast reconstruction: expectations for and impact on postmastectomy functioning. Psychosomatics. 1993;34:241-50.

28. Kitamura K, Ishida M, Inoue H, Kinoshida J, Hashizume M, Sugimachi K. Early results of an endoscope-assisted subcutaneous mastectomy and reconstruction for breast cancer. Surgery. 2002;131:S324-9.

29. Hung CS, Chang SW, Liao LM, Huang CC, Tu SH, Chen ST, et al. The learning curve of endoscopic total mastectomy in Taiwan: a multi-center study. PLoS One. 2017; e0183638. 